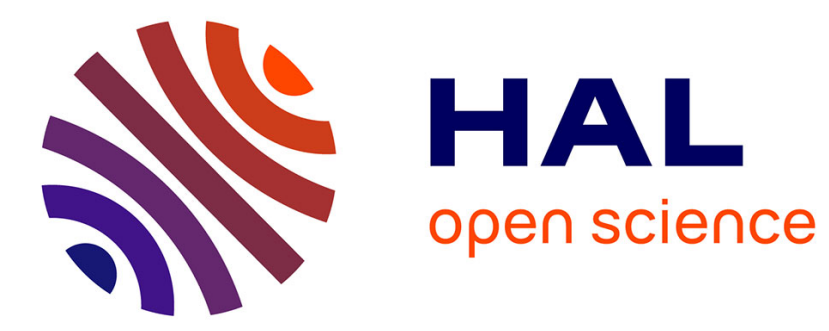

\title{
Observability Normal Forms for a class of switched systems with zeno phenomena
}

Lei Yu, Jean-Pierre Barbot, Driss Boutat, Djamila Benmerzouk

\section{To cite this version:}

Lei Yu, Jean-Pierre Barbot, Driss Boutat, Djamila Benmerzouk. Observability Normal Forms for a class of switched systems with zeno phenomena. 2009 American Control Conference, Jun 2009, St. Louis, Missouri, USA, United States. inria-00530116

\section{HAL Id: inria-00530116 https://hal.inria.fr/inria-00530116}

Submitted on 27 Oct 2010

HAL is a multi-disciplinary open access archive for the deposit and dissemination of scientific research documents, whether they are published or not. The documents may come from teaching and research institutions in France or abroad, or from public or private research centers.
L'archive ouverte pluridisciplinaire HAL, est destinée au dépôt et à la diffusion de documents scientifiques de niveau recherche, publiés ou non, émanant des établissements d'enseignement et de recherche français ou étrangers, des laboratoires publics ou privés. 


\title{
Observability Normal Forms for a class of switched systems with zeno phenomena
}

\author{
L. Yu, J.-P. Barbot, D. Boutat and D. Benmerzouk
}

\begin{abstract}
This paper deals with necessary and sufficient conditions to transform a class of switched systems to a particular form dedicated to observer design with and without zeno phenomena. Meanwhile, sufficient observability conditions for switched system with or without zeno phenomena are given. In the last section, some observer structures are proposed upon two academical examples.
\end{abstract}

Index Terms-Zeno phenomena, switched system, observability.

\section{INTRODUCTION}

$\mathbf{Z}$ Eno phenomenon is well known in physical domain, for example the bouncing ball case leading to many theoretical developments and simulations [2],[20]. In systems theory, the first order sliding mode [23] and high order sliding mode [14] [24] have been powerfully used in control design, as well as in observer design for many years. In control design problems, the main property of sliding mode is convergence of the system behavior in finite time under some matching condition [11] (respectively some observability condition [27] in observer design problems). Moreover, this property corresponds to a particular type of Zeno phenomena (i.e. chattering zeno [2] for the first order sliding mode). Based on this correspondence, this paper presents observability conditions for hybrid system with zeno phenomena by considering sliding mode and normal form approaches. These lead to two normal forms: one directly inspired on the observer matching condition and the other one on the Filippov theorem [16] and geometrical results [17]. For a sake of simplicity, we don't consider a switch on the output function and reset function at switching time.

Obviously, both observability forms presented in this paper can make sense only if there exist at least one observer for estimating the states when there exists zeno phenomena. Consequently, it is necessary to give some samples of building observers for each observability form presented in this paper. This paper is organized as follows: Section II gives the two proposed observability forms. Then conditions for the existence of diffeomorphism which transforms the system into one of the two normal forms are proposed in section III. Afterwards, sufficient observability conditions are presented

L.Yu is with Signal Processing Laboratory, Electronic and Information School, Wuhan University, China, and ECS ENSEA, 6 Avenue du Ponceau, 95014 Cergy-Pontoise, France. lei.yu@ensea. fr

J-P. Barbot is with ECS ENSEA, 6 Avenue du Ponceau, 95014 CergyPontoise, France. barbot @ensea. fr

D. Boutat is with LVR ENSI, 10 Boulevard de Lahitolle, 18020 Bourges, France. driss.boutateensi-bourges. fr

D. Benmerzouk is with Department of Mathematics, Tlemcen University, Algeria. d_benmerzouk@yahoo.fr

L.Yu and J-P. Barbot are with Projet Alien, INRIA, France. in section IV. In section V, conditions for the existence of diffeomorphism which transforms the system into one of the two normal forms for the linear hybrid system are proposed. In the last part, some observers are built for the two normal forms at the end of this paper.

\section{PRoblem STATEMENT}

Let us consider the following class of switched system:

$$
\begin{aligned}
\dot{x} & =f_{q}(x), \quad q \in\{1, \ldots, N\} \\
y & =h(x)
\end{aligned}
$$

where $x(t) \in U \subset \Re^{n}$ is the state, $y(t) \in \Re$ is the measured output and functions $f_{q}: U \rightarrow \Re^{n}, q \in Q$ and $h: U \rightarrow \Re$ are smooth for each $q . q$ is the discrete state which may be driven by a switching function $\sigma$, where $\sigma: \Re \rightarrow Q=\{1, \ldots, N\}$.

In order to deal with observability of systems (1), we will assume within this paper the following:

Assumption 1: For each $q \in Q$ the pair $\left(y, f_{q}\right)$ is regularly weakly locally observable ${ }^{1}$. Thus, $\operatorname{rank}\left\{d L_{f_{q}}^{j} h, \quad j=0\right.$ : $n-1\}=n$.

Hereafter we give two normal forms, each of them corresponds to a particular hybrid observability form and different assumptions on the discrete state are requested in order to consider state observation with zeno phenomena.

\section{A. First observability form}

The first proposed normal form gives the following form:

$$
\begin{aligned}
\left(\begin{array}{c}
\dot{\xi}_{1} \\
\dot{\xi}_{2} \\
\dot{\xi}_{3} \\
\vdots \\
\dot{\xi}_{n}
\end{array}\right) & =\left(\begin{array}{c}
0 \\
\xi_{1} \\
\xi_{2} \\
\vdots \\
\xi_{n-1}
\end{array}\right)+\left(\begin{array}{c}
\tilde{f}_{q} \\
0 \\
0 \\
\vdots \\
0
\end{array}\right) \\
y & =\tilde{h}(\xi)=\xi_{n}
\end{aligned}
$$

where $q \in Q$ is the discrete state.

Remark 1:

$1-)$ It is clear from the form (3)-(4) that the observation of the state $\xi$ is independent with respect to the discrete state $q$.

$2-$ ) In the form (3)-(4) the function $\tilde{f}_{q}(\xi)$ may be considered as a perturbation, consequently it is not surprising that the necessary and sufficient conditions to transform the original

\footnotetext{
${ }^{1}$ At the classical locally weakly observability [19], it is added the regularity of the first $n-1$ derivatives.
} 
system in this form are similar to the observability matching condition [27].

\section{B. Second observability form}

The second case considered in the next section is to exhibit sufficient and necessary conditions for the existence of diffeomorphism $z=\phi(x)$ such that the system (1)-(2) is transformed into the following form:

$$
\begin{aligned}
\dot{z} & =\alpha_{0}(z)+\gamma_{q}(y), \quad q \in Q \\
y & =z_{n}
\end{aligned}
$$

Remark 2: The local weakly observability property of system (5-6) doesn't depend on the term of $\gamma_{q}(y)$. Thus, it is equivalent to the observability of the pair $\left(\alpha_{0}(z), z_{n}\right)$.

\section{MAIN RESULTS}

In this section, the structural conditions is analyzed in order to transform by diffeomorphism system (1)-(2) into one of both cases previously introduced. Here the difference between two functions is considered:

$$
f_{\Delta_{\kappa, \nu}}(x)=f_{\kappa}(x)-f_{\nu}(x), \quad \kappa, \nu \in Q
$$

And this function will be used in the analysis of both case.

\section{A. Condition for the existence of a diffeomorphism in the} first case

Hereafter, sufficient and necessary condition are given for the existence of diffeomorphism $\xi=\psi(x)$ such that system (1)-(2) is transformed into the form (3)-(4). Moreover, the proof of the next proposition is a constructive one.

Proposition 1: Under assumption 1, there exist a diffeomorphism $\xi=\psi(x)$ which transforms the system (1)-(2) into the form (3)-(4) if and only if the following conditions are fulfilled:

$$
L_{f_{\Delta_{\kappa}, \nu}} L_{f_{\kappa}}^{j-1} h=0 \forall \kappa, \nu \in Q \text { and } \forall j \in\{1, \ldots n-1\}
$$

Proof: Necessity: the form (3)-(4) satisfy assumption 1 and condition (7).

Sufficiency: setting $z_{n}=\psi_{n}=h$, condition (7) gives $\left(L_{f_{\kappa}} h=L_{f_{\nu}} h\right)$ and assumption 1 implies that $d h, d L_{f_{\kappa}} h$ are linearly independent, consequently it is possible to define $z_{n-1}=L_{f_{\kappa}} h=\psi_{n-1}$. Finally by induction the diffeomorphism is defined as follow $z_{n-i}=L_{f_{\kappa}}^{i} h$ for $i \in\{1, \ldots n-1\}$.

Remark 3: If $f_{\Delta_{\kappa, \nu}}$ is not trivial then assumption 1 and condition (7) imply that $L_{f_{\Delta_{\kappa}, \nu}} L_{f_{\kappa}}^{n-1} h \neq 0$. Note that, this condition is closed to the notion of relative index [26] and observability matching condition [27]. Moreover, under this condition and for a switch $\sigma$ without Zeno we can estimate the discrete state $q$. (For more details see [6][15]).

Remark 4: From the observability concept introduced by M. Fliess and S. Diop [13] in the theoretical frames of differential algebra, proposition 1 is equivalent to the existence of a common function $F$ ( $F$ is independent to $q$.), for all subsystems, which verifies the following algebraic equation:

$$
x=F\left(y, \ldots, y^{(n-1)}\right)
$$

where $y^{(j)}$ denotes the $j^{\text {th }}$ derivative of $y$.
B. Condition for the existence of a diffeomorphism in the second case

Hereafter, we will exhibit sufficient and necessary conditions for the existence of the diffeomorphism for the second case $\phi$, which transforms the system (1)-(2) into the system (5)-(6).

Theorem 1: Under assumption 1, sufficient and necessary conditions for the existence of diffeomorphism $\phi(x)$ such that the system (1)-(2) is transformed into system (5)-(6) are:

There exist a family of independent vector fields $\left\{\tau_{1}, \ldots \tau_{n}\right\}$ such that:

$$
\begin{array}{ll}
\text { a)- } & d h \cdot \tau_{n}=1 \\
\text { b)- } & d h \cdot \tau_{i}=0, \forall i \in\{1, \ldots, n-1\} \\
\text { c)- } & {\left[\tau_{i}, \tau_{j}\right]=0, \forall(i, j) \in\{1, \ldots, n\} \times\{1, \ldots, n\}} \\
d)-\quad & {\left[\tau_{i}, f_{\Delta_{\kappa, \nu}}\right]=0, \forall i \in\{1, \ldots, n-1\}, \forall \kappa, \nu \in Q} \\
\text { Proof: } &
\end{array}
$$

1) Sufficiency: As the family $\left\{\tau_{i}\right\}_{1 \leq i \leq n}$ is a basis of the tangent fibre bundle $T U$ of $U$ and thanks to condition $c$ ) there exists a change of coordinates $z=\phi(x)$ such that the differential function of $\phi$ satisfies:

$$
\phi_{*}\left(\tau_{i}\right)=\frac{\partial}{\partial z_{i}} \quad \text { for all } i \in\{1, \ldots, n\} .
$$

Now we will calculate $f_{\Delta_{\kappa, \nu}}$ in the $z$ coordinates, from $d$ ) and the linearity of $\phi_{*}$ we have for $i \in\{1, \ldots, n-1\}$ :

$$
\frac{\partial}{\partial z_{i}}\left(\phi_{*}\left(f_{\Delta_{\kappa, \nu}}\right)\right)=\left[\phi_{*}\left(\tau_{i}\right), \phi_{*}\left(f_{\Delta}\right)\right]=0,
$$

which implies that:

$$
\phi_{*}\left(f_{\Delta_{\kappa, \nu}}\right)=\sum_{i=1}^{n} \varphi_{i}\left(z_{n}\right) \frac{\partial}{\partial z_{i}}
$$

Conditions $a$ ) and $b$ ) mean that $z_{n}=y$. Therefore there exist functions $\varphi_{i}^{\kappa, \nu}(y)_{1 \leq i \leq n}$ on $U$ such that:

$$
f_{\Delta_{\kappa, \nu}}=\sum_{i=1}^{n} \varphi_{i}^{\kappa, \nu}(y) \tau_{i}
$$

2) Conditions $a), b), c)$ and $d$ ) are necessary. In fact, assume that:

$$
f_{\Delta_{\kappa, \nu}}(x)=\sum_{i=1}^{n} \varphi_{i}^{\kappa, \nu}(y) \frac{\partial}{\partial x_{i}} \quad \text { and } \quad y=x_{n} .
$$

then the seek base is $\tau_{i}=\frac{\partial}{\partial x_{i}}$ for $i \in\{1, \ldots, n\}$.

Remark 5: A class of dynamical systems which fulfill the conditions of theorem 1 are the dynamical systems, linearizable by the same diffeomorphism and output injection, which are characterized by the theorem of Krener and Isidori [17].

Here after we will give an example of dynamical system which satisfies the theorem 1, however, it doesn't satisfy the theorem of Krener and Isidori [17]. This means that the class which satisfies the theorem 1 is larger than the class which satisfies the theorem of Krener and Isidori, this is obviously due to the fact that we avoid a linear condition for $\alpha_{0}$.

Example 1: Consider this dynamical example, 


$$
\begin{aligned}
\left(\begin{array}{l}
\dot{x}_{1} \\
\dot{x}_{2}
\end{array}\right) & =\left(\begin{array}{c}
-x_{1}\left(x_{2}-x_{1}\right) \\
x_{1}\left(x_{1}-x_{2}-1\right)
\end{array}\right)+\left(\begin{array}{c}
\left(x_{2}-x_{1}\right)^{2} \\
0
\end{array}\right) \\
y & =x_{2}-x_{1} \\
\left(\begin{array}{c}
\dot{x}_{1} \\
\dot{x}_{2}
\end{array}\right) & =\left(\begin{array}{c}
-x_{1}\left(x_{2}-x_{1}\right) \\
x_{1}\left(x_{1}-x_{2}-1\right)
\end{array}\right)+\left(\begin{array}{c}
2\left(x_{2}-x_{1}\right)^{2} \\
0
\end{array}\right) \\
y & =x_{2}-x_{1}
\end{aligned}
$$

Now after assemble calculations, the vector fields $\tau$ are given by $\tau_{1}=\left(\begin{array}{l}1 \\ 1\end{array}\right)$ and $\tau_{2}=\left(\begin{array}{l}0 \\ 1\end{array}\right)$.

From system (12), the difference between the associated two dynamical functions can be obtained as $f_{\Delta}\left(x_{1}, x_{2}\right)=$ $\left(\begin{array}{c}-\left(x_{2}-x_{1}\right)^{2} \\ 0\end{array}\right) \cdot{ }^{2}$ By some calculation, the condition a) and b) can be easily verified. And the condition c) is obvious since the vector fields $\tau_{1}, \tau_{2}$ are with constant values. For condition d), $\left[\tau_{1}, f_{\Delta}\right]=L_{\tau_{1}} f_{\Delta}=0$.

Through the following diffeomorphism

$$
\left(\begin{array}{l}
\xi_{1} \\
\xi_{2}
\end{array}\right)=\phi(x)=\left(\begin{array}{c}
x_{1} \\
x_{2}-x_{1}
\end{array}\right)
$$

The switched system (12) can be transformed as

$$
\begin{aligned}
&\left(\begin{array}{c}
\dot{\xi}_{1} \\
\dot{\xi}_{2}
\end{array}\right)=\left(\begin{array}{c}
-\xi_{1} \xi_{2} \\
-\xi_{1}
\end{array}\right)+\left(\begin{array}{c}
\xi_{2}^{2} \\
-\xi_{2}^{2}
\end{array}\right) \\
& y=\xi_{2} \\
&\left(\begin{array}{c}
\dot{\xi}_{1} \\
\dot{\xi}_{2}
\end{array}\right)=\left(\begin{array}{c}
-\xi_{1} \xi_{2} \\
-\xi_{1}
\end{array}\right)+\left(\begin{array}{c}
2 \xi_{2}^{2} \\
-2 \xi_{2}^{2}
\end{array}\right) \\
& y=\xi_{2}
\end{aligned}
$$

Remark 6: A necessary condition for the existence of diffeomorphism $\phi(x)$ such that the system (1)-(2) is transformed into system (5)-(6) is:

$$
\operatorname{Rank}\left(\begin{array}{c}
\frac{\partial f_{\Delta}}{\partial x} \\
\frac{\partial h}{\partial x}
\end{array}\right)=\operatorname{Rank}\left(\frac{\partial h}{\partial x}\right)=1,
$$

on $U$.

Remark 7: It is important to mention that the previous theorem gives a necessary and sufficient conditions for transforming the system (1)-(2) into the system (5)-(6) but it is necessary to introduce also some extra conditions on the knowledge of the state $q$ (or equivalently $\sigma$ ) in order to be able to design an observer.

\section{SUFFICIENT OBSERVABILITY CONDITIONS}

\section{A. Case One}

For system of the form (3)-(4) algebraic estimator [3], [21] or step by step sliding mode observer [4], [15] work well in the continuous state estimation, because in the first equation of (3), $\dot{\xi}_{1}$ is never considered.

More precisely, the output derivative is considered only until $n-1$ in algebraic solution and the last step is a sliding mode observer of one in the step by step sliding mode observer, i.e. it is requested that $\dot{z}_{1}$ is bounded and the bound is known.

\footnotetext{
${ }^{2}$ When the switched system is only composed of two subsystems, then $f_{\Delta_{1,2}}$ can be abbreviate to $f_{\Delta}$
}

From these and proposition 1, it is possible to set the following corollary:

Corollary 1: Under assumption 1, a sufficient condition for the observability of the continuous state of system (5)(6) with zeno phenomena is:

$$
L_{f_{\Delta_{\kappa}, \nu}} L_{f_{\kappa}}^{j-1} h=0 \forall \kappa, \nu \in Q \text { and } \forall j=\{1, \ldots, n-1\}
$$

\section{B. Case two}

In the form (5)-(6), the discrete state $q$ is not considered as a perturbation, consequently, the following assumption is requested:

Assumption 2: The discrete state $q$ is known.

Obviously, this assumption is practically impossible to be guarantied in the case of zeno phenomena, such as for the Chattering Zeno (i.e. after some time the dwell time is exactly equal to zero) or for the Genuinely Zeno (i.e. the dwell time is never equal to zero)[2]. So assumption 2 is replaced by the following one:

Assumption 3:

a- The discrete state $q$ is Henstock-Kurzweil-Pettits integrable $^{3}[18][8][28]$ or NV-Integrable[12] and its averages is measured via a low pass filter of sufficiently large bandwidth on time interval $[0, \alpha]$

b- The system (1)-(2) is affine with respect to the discrete state $q$.

The second condition of the assumption above and the conditions of theorem 1 imply that there exists a diffeomorphism such that system (1)-(2) can be transformed into:

$$
\begin{aligned}
\dot{z} & =\alpha_{0}(z)+\gamma(y) q \\
y & =\tilde{h}(z)=z_{n}
\end{aligned}
$$

So practically, it is only possible to obtain the filtered discrete state $q_{f}$ (instead of the real discrete state $q$ ).

Now, let us consider the system (5)-(6) reduced to the common dynamics:

$$
\begin{aligned}
\dot{z} & =\alpha_{0}(z) \\
y & =\tilde{h}(z)=z_{n}
\end{aligned}
$$

and assuming:

Assumption 4: For the system (15)-(16), there exist an observer such that the continuous state observation error (i.e. the difference between the continuous state ant its estimate) is exponentially stable.

Remark 8: Under some specific assumptions as Lipschitz condition, persistent excitation,.. it is possible to use classical high gain observer [5] or adaptive observer [7] For these observer classes, the exponential stability of the continuous state observation error is guarantied.

From the previous assumption and theorem 1, it is possible to set the following proposition:

Proposition 2:

- - A) Assumptions 1, 2, 4 and conditions of the theorem 1 are sufficient conditions for the continuous state

\footnotetext{
${ }^{3}$ see also Denjoy-Khinchine integrable.
} 
observability ${ }^{4}$ of system (1)-(2) with zeno phenomena. - - B) Assumptions 1, 3, 4 and conditions of theorem 1 are sufficient conditions for the practical $^{5}$ continuous state observability of the system (1)-(2) with zeno phenomena.

Proof: Proof of the part $A$ of the proposition: there exists an observer of the form:

$$
\begin{aligned}
\dot{\hat{z}} & =\beta(\hat{z}, y, \hat{y}) \\
\hat{y} & =\hat{z}_{n}
\end{aligned}
$$

which ensures the exponential stability of the continuous state observation error $e=z-\hat{z}$. Consequently, there exists a Lyapunov function $V(e)$ with respect to $(15),(16)$ and (17),(18) such that:

$$
\dot{V}=\frac{\partial V}{\partial e}\left(\alpha_{0}(z)-\beta(\hat{z}, y, \hat{y})\right)<-K V
$$

with $K>0$.

Modifying (17),(18) as follow:

$$
\begin{aligned}
\dot{\hat{z}} & =\beta(\hat{z}, y, \hat{y})+\gamma_{q}(y) \\
\hat{y} & =z_{n}
\end{aligned}
$$

The state observation error for system (5)-(6) and observer (20)-(21) is exponentially stable, because the previous Lyapunov function in this case gives:

$$
\dot{V}=\frac{\partial V}{\partial e}\left(\alpha_{0}(z)-\beta(\hat{z}, y, \hat{y})+\gamma_{q}(y)-\gamma_{q}(y)\right)<-K V
$$

Proof of the part $B$ : the observation error becomes:

$$
\dot{e}=\alpha_{0}(z)-\beta(\hat{z}, y, \hat{y})+\gamma(y)\left(q-q_{f}\right)
$$

From condition a) of the assumption 3, it can be obtained that

$$
\dot{p}=\gamma(y)\left(q-q_{f}\right)
$$

which is Cauchy problem in the sense of Henstock-KurzweilPettis integrals. Using the same method as Filippov in [16] page 17 , it is possible to set $\epsilon=e-p$ and it can be obtained that

$$
\dot{\epsilon}=\alpha_{0}(z)-\beta(\hat{z}, y, \hat{y})
$$

which admit a local solution in the framework of Carathodory for $t \in[0, \Gamma] \subset[0, \alpha]$. Moreover, the assumption 4 and the observer (17)-(18) ensure that there exist a Lyapunov function $V(e)$ for (15)(16), which verifies (19). Consequently, derivation of $V(\epsilon)$ with respect to (24), gives

$$
\dot{V}(\epsilon)=\frac{\partial V}{\partial \epsilon}\left(\alpha_{0}(z)-\beta(\hat{z}, y, \hat{y})\right)
$$

As $\epsilon=e-p$, it implies that $\left.\frac{\partial V}{\partial \epsilon}\right|_{\epsilon}=\left.\frac{\partial V}{\partial e}\right|_{\epsilon}$ and from the assumption 4 , it is possible to rewrite the equation (25) as follows

$$
\begin{aligned}
\dot{V}(\epsilon)= & \left.\frac{\partial V}{\partial e}\right|_{e}\left(\alpha_{0}(z)-\beta(\hat{z}, y, \hat{y})\right) \\
& -\left.\frac{1}{2} \frac{\partial^{2} V}{\partial e^{2}}\right|_{e}\left[O(p) \otimes\left(\alpha_{0}(z)-\beta(\hat{z}, y, \hat{y})\right)\right]
\end{aligned}
$$

\footnotetext{
${ }^{4}$ The observation error can be assign in any measurable vicinity of zero

${ }^{5}$ The observation error must be as small as it can be but not zero.
}

with $\lim _{p \rightarrow 0} O(p)=0$. And (19) gives

$$
\begin{aligned}
\dot{V}(\epsilon) & \leq-K V(\epsilon) \\
& +\left|\frac{1}{2} \frac{\partial^{2} V}{\partial e^{2}}\right|_{e}\left[O(p) \otimes\left(\alpha_{0}(z)-\beta(\hat{z}, y, \hat{y})\right)\right] \mid(26)
\end{aligned}
$$

As for all $\epsilon>0$, there exists a filter and $t_{1} \geq 0$ such that $\forall t>t_{1}$, we have $\|p\|=\sup _{0 \leq t \leq \Gamma}\left|\int_{t_{1}}^{t} \gamma(y)\left(q-q_{f}\right)\right|<\varepsilon$. Consequently, it is possible, for each $V_{d}>0$ to set $\varepsilon<<\frac{V_{d}}{2}$ and the inequality (26) becomes

$$
\dot{V}(\epsilon) \leq-K V+|O(\varepsilon)|
$$

So, one can conclude that for $e \notin E_{V_{d}}:=\left\{e / V(e)<V_{d}\right\}$, such that

$$
\dot{V}(\epsilon) \leq-\frac{K}{2} V(\epsilon)
$$

Because, $\varepsilon<<\frac{V_{d}}{2}$ guaranties that $|O(\varepsilon)|<\frac{V_{d}}{2}$.

\section{LINEAR VIEWPOINT}

For linear case, system (1)-(2) would be written as

$$
\begin{aligned}
\dot{x} & =A_{q} x, \quad q \in\{1, \ldots, N\} \\
y & =C x
\end{aligned}
$$

where $A_{q}$ is a $n \times n$ matrix and $C$ is an $n \times 1$ vector.

\section{A. Condition for the first case}

The sufficient and necessary condition for the existence of linear diffeomorphism $\xi=\Phi x$ such that system (27)(28) can be transformed into the form (3)-(4) is given by,

Proposition 3: Under assumption 1, there exists a linear diffeomorphism $\xi=\Phi x$ which transforms the system (27)(28) into the form (3)-(4) if and only if the following condition is fulfilled:

- $C A_{\kappa}^{j}=C A_{\nu}^{j}$, where $j=1, \ldots, n-1, \kappa, \nu \in Q$

Proof: Necessity:

System (3)-(4) shows that for each $i \in\{1, \ldots, n-1\}$, it is satisfied

$$
y_{\kappa}^{(i)}=y_{\nu}^{(i)}
$$

this means that the $i$-th derivative is independent from the discrete state $q$, i.e. the necessity of the condition is verified.

Sufficiency:

Construct a diffeomorphism as

$$
\xi_{k}=C A_{q}^{n-k}
$$

where $k \in\{1, \ldots, n-1\}$ and $q=\kappa$ or $q=\nu$. Then, we can easily verify the sufficiency of condition. 


\section{B. Condition for the second case}

The sufficient and necessary condition for the existence of linear diffeomorphism $z=\Psi x$ such that system (27)-(28) can be transformed into the form (5)-(6) is given as,

Proposition 4: Under assumption 1, a sufficient and necessary condition for the existence of linear diffeomorphism $z=\Psi x$ such that the system (27)-(28) is transformed into system (5)-(6) is that there exist a family of independent vectors $\left\{\tau_{1}, \ldots, \tau_{n}\right\}$ such that:

a) $C \cdot \tau_{n}=1$;

b) $C \cdot \tau_{i}=0$, with $i \in 1, \ldots, n-1$;

c) $\left(A_{\kappa}-A_{\nu}\right) \cdot \tau_{i}=0$, with $i \in 1, \ldots, n-1$, and $\forall \kappa, \nu \in Q$. Proof: a) Sufficiency:

Because the vectors $\left\{\tau_{1}, \ldots, \tau_{n}\right\}$ are independent, there exists a diffeomorphism which is $z=\Psi x$, where $\Psi^{-1}=$ $\operatorname{col}\left\{\tau_{1}, \ldots, \tau_{n}\right\}$.

So, consider the following system

$$
\dot{x}=\frac{A_{\kappa}+A_{\nu}}{2} x+H_{q} \frac{A_{\kappa}-A_{\nu}}{2} x
$$

where $H_{q}=\left\{\begin{array}{c}-1 \text { for } q=\kappa \\ 1 \text { for } q=\nu\end{array}\right.$.

And rewrite the first derivative of $z$ with the system above

$$
\begin{aligned}
\dot{z} & =\Psi \dot{x} \\
& =\Psi \frac{A_{\kappa}+A_{\nu}}{2} \Psi^{-1} z+H_{q} \Psi \frac{A_{\kappa}-A_{\nu}}{2} \Psi^{-1} z
\end{aligned}
$$

From condition $(a)-(b)$, we obtain that $y=C x=z_{n}$. And from condition (c), $H_{q} \Psi \frac{A_{\kappa}-A_{\nu}}{2} \Psi^{-1} z=B_{q} z_{n}=B_{q} y$.

b) Necessity:

Suppose there exists a diffeomorphism $z=\Psi x$, then

$$
\begin{aligned}
\dot{z} & =\Psi \dot{x} \\
& =\Psi \frac{A_{\kappa}+A_{\nu}}{2} \Psi^{-1} z+H_{q} \Psi \frac{A_{\kappa}-A_{\nu}}{2} \Psi^{-1} z
\end{aligned}
$$

In fact $\operatorname{col}\left\{\tau_{1}, \ldots, \tau_{n}\right\}=\Psi^{-1}$, then the condition $(a)-(c)$ can be easily verified.

\section{Simulations}

The objective of this section is to highlight the efficiencies of these observability conditions. Moreover, some observers for each system with zeno phenomena are given. To make the simulations more general, three observer structures are adapted, ALIEN observer [21][3][22][9], the second order sliding mode observer[23][15][24], and the high-gain observer[5].

\section{A. Example for the first observability form}

Let us consider the very simple academical system in the form (3)-(4)

$$
\begin{aligned}
\dot{z}_{1} & =f_{q}\left(z_{1}, z_{2}\right), \quad \dot{z}_{2}=z_{1} \\
y & =z_{2}
\end{aligned}
$$

with $f_{q}=-z_{1}-2 z_{2}+q$ and $q=\operatorname{sign}(w)$ where $w=N$ is a white noise. This form of $q$ theoretically leads to the Zeno phenomenon. For this system, we apply the ALIEN observer and the second order sliding mode observer to estimate $z_{1}$,
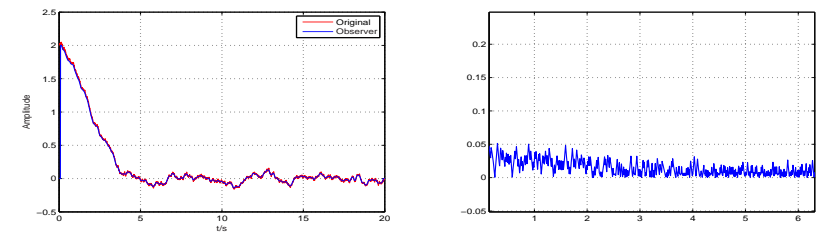

Fig. 1. (Left)Result of ALIEN Observer without noise; (Right)Corresponding Error.
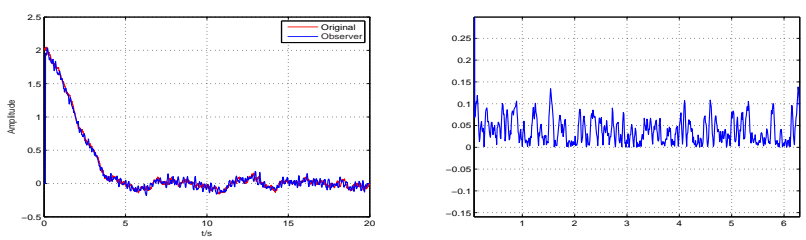

Fig. 2. (Left)Result of ALIEN Observer with noise; (Right)Corresponding Error.

one state of system (30)-(31). ALIEN algorithm is numerical differential algebraic approach, proposed by M. Fliess et al. in [3], and roughly speaking, the main fundamental principle of this method is the derivative of Laplace transform with respect to $s$ starting from Taylor expansion in the time domain of the original signal. In this paper, we use the individual estimation algorithm [3].

Meanwhile, the second order sliding mode observers are built for the continuous states[23] [15] [24]. Fig 1, 3 are the results of ideal condition without any noise in the measurement, and Fig 2, 4 are the results of observers with noisy measurements, which makes the simulation a little more realistic, and the power of the noise are assigned to $E_{n o i s e}=0.01$. The left figures of Fig 1,2,3,4 are the results of observer and the right are the difference between observer and original value of the state. Obviously, the state $z_{1}$ can be observed from these observer structures, and from the error plot, defined as $e_{\text {erro }}=\left|\hat{z}_{1}-z_{1}\right|$, the performance of the observer structures can be considered satisfied.

\section{B. Example for second observability form}

Let us consider another very simple academical system in the form (5)-(6)

$$
\begin{aligned}
\dot{z}_{1} & =-z_{2}, \quad \dot{z}_{2}=z_{1}+\left(-z_{2}^{3}+q\right) \\
y & =z_{2}
\end{aligned}
$$
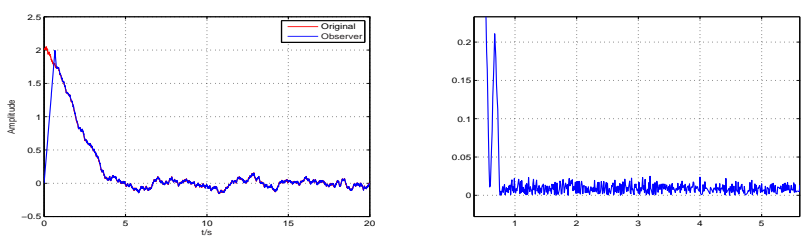

Fig. 3. (Left)Result of second order sliding mode observer without noise; (Right)Corresponding Error. 

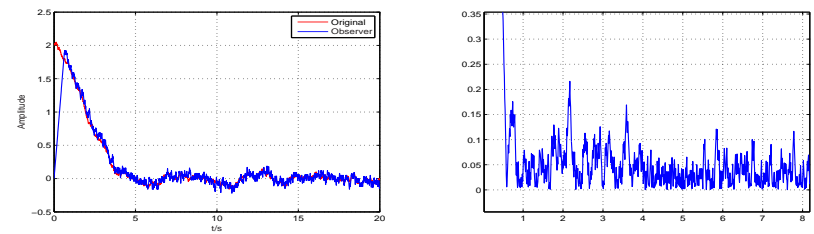

Fig. 4. (Left)Result of second order sliding mode observer with noise; (Right)Corresponding Error.

with $q=1.5+0.5 \operatorname{sign}(w)$ and $w=N+e^{-t}$, where $N$ is a white noise. This form of $q$ theoretically leads to infinite commutation in finite time.

In this case, due to assumption 4, high-gain observer is adapted.

For this system (32)-(33), the high-gain observer is designed as follows

$$
\dot{\hat{z}}=A_{0} \hat{z}+\phi(\hat{z})-\Lambda(\lambda)^{-1} K_{0}\left(C_{0} \hat{z}-y\right)
$$

where $A_{0}=(0,-1 ; 1,0), C_{0}=(0,1)$ and $\phi((\hat{z}))=$ $\left(0,-\hat{z}_{2}^{3}+q_{f}\right)^{T}$. Here, we choose $\lambda=10, K_{0}=(0,100)^{T}$ and $\Lambda(\lambda)^{-1}=\operatorname{diag}(1, \lambda)$. The results are shown in Fig 5 without noise in the measurement, and Fig 6 with noise in the measurement. Obviously in both cases the observers work well.
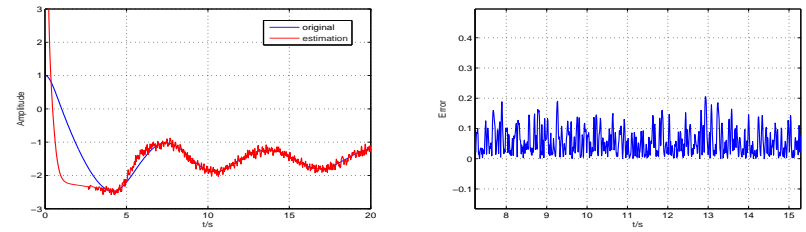

Fig. 5. (Left)Result of High-Gain Observer without noise; (Right)Corresponding Error
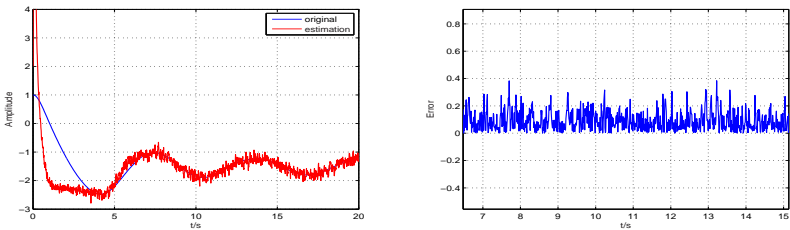

Fig. 6. (Left)Result of High-Gain Observer with noise; (Right)Corresponding Error.

Above all, we can make a conclusion that it is possible to observe a hybrid system under specific conditions even if zeno phenomenon occurs. Obviously this work considers a very primary case and many other forms and cases should be considered. One of the most difficult problem for us is to observe hybrid systems with jumps under Zeno phenomenon (i.e. the case of bouncing ball).

\section{REFERENCES}

[1] L. Yu, J.-P. Barbot, D. Boutat, D. Benmerzouk. Formes Normales d'Observabilité et Phénomène de Zénon, Conférence Internationale Francophone d'Automatique, CIFA2008, Bucarest, Roumanie.

[2] A. Ames, H. Zheng, R. Gregg, S. Sastry, Is there life after Zeno? Taking executions past the breaking (zeno) point, IEEE ACC, Mineapolis, 2006

[3] M. Mboup, C. Join, M. Fliess, A revised look at numerical differentiation with an application to nonlinear feedback control, The $15^{\text {th }}$ Mediterrean Conference on Control and Automation - MED'2007 (2007)

[4] J.-P. Barbot, T. Boukhobza, M. Djemaï, Sliding mode observer for triangular input form, IEEE Conf. Decision Control, Kobe, 1996.

[5] G. Besançon, Q. Zhang, H. Hammouri, High-Gain Observer based State and Parameter Estimation in Nonlinear System, 6th IFAC symposium on Nonlinear Control Systems, 2004.

[6] D. Boutat, A. Benali and J.-P. Barbot, About the observability of piecewise dyamical system, IFAC NOLCOS'2004.

[7] K. Busawon and J. de Leon Morales, An observer design for uniformly observable nonlinear systems, Int. J. of Control, Vol. 73, No. 15, pp. 1357-1381. 2000

[8] M. Cichon, I. Kubiaczyk and A. Sikorska, The henstock-KurweilPettis integral and exsitence theorems for the Cauchy problem, Czeckoslovak Mathematical Journal, Vol 54, pp 279-289, 2004.

[9] J. Daafouz, M. Fliess, G. Millerioux, Une approche intrinsèque des observateurs linéaires à entrées inconnues, Actes Conf. Internat. Automat. Francoph. - CIFA 2006, Bordeaux, 2006

[10] S. Drakunov and V. Utkin, "Sliding mode observer: Tutorial", in Proc. of the 34th IEEE CDC95, 1995.

[11] B. Drazenovic, The invariance condition in variable structure systems, Automatica, Vol 5, No.3, pp 287-295, 1969.

[12] S. De Fogio, The NV-Integrals, AMS Special Session on Nonabsolute Integration, Toronto, 2000.

[13] S. Diop, M. Fliess, On nonlinear observability, Proc. $1^{\text {st }}$ Europ. Control Conf., Hermès, pp. 152-157, 1991.

[14] S. V. Emelyanov and S. K. Korovin, Applying the principle of control by deviation to extend the set of possible feedback types, Soviet Physics, Doklady, Vol 26 No. 6, pp.562-564, 1981.

[15] T. Floquet, J.-P. Barbot, Super twisting algorithm-based step-bystep sliding mode observers for nonlinear systems with unknown inputs, International Journal of Systems Science, Volume 38, Issue 10 January 2007 , pages $803-815$.

[16] A. F. Filippov, Differential Euations with Discontinuous Righthand sides, Kluwer Academic Publishers, 1988.

[17] A.J. Krener and A. Isidori, Linearization by output injection and nonlinear observer, Sys. \& Control Letters, vol.3, pp. 47-52, 1983.

[18] R. Henstock, The General Theory of Integration, Oxford Mathematical Monographs, Clarendon Press, Oxford 1991.

[19] R. Hermann, A. J. Krener, Nonlinear controllability and observability, IEEE Transactions on Automatic Control, Vol. 22, pp. 728-740, 1977.

[20] M. Heymann, F. Lin, G. Meyer and S. Resmerita, Analysis of Zeno behaviours in hybrid systems, In Proc. of 41th IEEE CDC, Las Vegas, 2002.

[21] M. Mboup, Parameter estimation via differential algebra and operational culculus, Rapport de recherche 40 (2007)

[22] J.-P. Barbot, M. Fliess, T. Floquet, An algebraic framework for the design of nonlinear observers with unknown inputs, $46^{\text {th }}$ IEEE Conference on Decision and Control (2007)

[23] V.I.Utkin, Sliding Modes in Optimization and Control, SpringerVerlag, 1992.

[24] A. Levant, Sliding order and sliding accuracy in sliding mode control, Int. J. of Control, Vol. 58, No. 6, pp. 1253-1253, 1993.

[25] A. Levant, Robust Extract Differentiation via Sliding mode Technique, Automatica, Vol. 34, No. 3, pp. 379-384, 1998.

[26] A. Isidori, Nonlinear Control Systems, Communication and Control Engineering Series, Third edition, Springer-Verlag, 1995.

[27] W. Perruquetti and J.-P. Barbot, Sliding Mode Control in Engineering, Ed. Marcel Dekker, 2002.

[28] E. Talvila, Estimates of the remainder in Taylor's theorem using the Henstock, Czeckoslovak Mathematical Journal, Vol.55, pp. 993-940, 2005. 